\title{
Phase behaviour of very asymmetric binary mixtures
}

\author{
José A Cuesta and Yuri Martínez-Ratón† \\ Grupo Interdisciplinar de Sistemas Complicados, Departamento de Matemáticas, Universidad \\ Carlos III de Madrid, 28911-Leganés, Madrid, Spain
}

\begin{abstract}
The phase behaviour of very asymmetric binary mixtures can be understood in terms of the depletion interaction. For hard particles this yields a narrow deep attractive well surrounding the hard core. Colloids with similar interaction potentials are known to destabilize the liquid, causing it to show a wide fluid-solid coexistence, and in extreme cases they exhibit an exotic solid-solid condensation. For a mixture this means that phase separation is not fluid-fluid, as previously thought, but normally fluid-solid, and if the asymmetry is very large, even solid-solid. We present in this work the result of devising a density functional theory for an infinitely asymmetric mixture of parallel hard cubes. This model is singular and undergoes a collapse in a close-packed solid (an extreme fluid-solid demixing). We avoid this collapse by introducing a small amount of polydispersity in the large particles; the resulting phase diagram shows the fluid-solid and solid-solid demixing scenarios described above.
\end{abstract}

When we have a system with more than one microscopic component (a mixture), the system can exist as a single, uniform phase, or it can undergo a phase transition and separate into two or more phases, each with a different composition. This phase transition is commonly referred to as demixing. One can think of a simple microscopic mechanism by which demixing can occur. For the sake of simplicity let us restrict consideration to binary mixtures. If unlike particles attract each other more weakly (or even repel each other) than like particles do, then in view of the simple balance between energy and entropy it is conceivable that, for certain compositions, below some temperature the system demixes.

Less obvious, but still feasible, is the entropic mechanism. This is illustrated by mixtures of hard particles. Suppose (as happens for certain mixtures of atomic particles) that particles of different species 'see' each other as having larger diameters. In this case there is more free volume if the system is phase separated than if it is mixed. If this gain compensates for the entropy lost by ordering (and it does for sufficient non-additivity), then demixing occurs [1,2]. The effect is made beautifully clear in a lattice model introduced some years ago by Frenkel and Louis [3], in which two different types of particle coexist in a square lattice. A transformation of the model exactly maps it to the Ising model with an external field, hence unambiguously proving the existence of entropic demixing.

One way of looking at the entropic mechanism of demixing is in the depletion picture introduced long ago by Asakura and Oosawa [4]. In a mixture of 'big' and 'small' particles (provided that this distinction is sufficiently clear) the big ones experience an effective attraction if two of them are so close to each other that no small particle fits in between them. The

$\dagger$ Current address: Fachbereich Physik, Bergische Universität Wuppertal, Gaußstraße 20, Wuppertal D-42097, Germany. 
unbalanced osmotic pressure that the small particles exert on the big ones produces a net attractive force.

An interesting question concerning the entropic demixing is that of how much nonadditivity the system must have in order to undergo demixing. For a long time it was believed that some non-additivity was needed, because the solution to the Ornstein-Zernike equation, in the Percus-Yevick approximation, for a binary mixture of hard spheres predicts its stability for any composition and diameter ratio [5]. At the beginning of the 1990s, using more accurate closure approximations, several authors [6-8] showed strong evidence supporting the existence of a spinodal instability for this system, provided that the diameter ratio is smaller than $0.2-0.1$ (depending on the author). Such asymmetric mixtures are very difficult to simulate (the probability of a big particle overlapping a small one in a Monte Carlo move is so high at the packing fractions of interest that big particles can hardly be moved). Only with the help of tricky cluster moves was the same evidence found in a simulation [9].

At the same time, experiments carried out on suspensions of polystyrene or silica spheres $[10,11]$ showed conclusive evidence of phase separation in asymmetric mixtures of hard spheres, but while one phase remained fluid (the one rich in small particles) the other one always formed a crystal. This fact was later confirmed by some theories [12,13]; however, the final scenario is a little more subtle.

In order to understand the physics of binary mixtures it is convenient to shift to the 'depletion picture'. The idea consists in mapping the mixture to an effective fluid in which the big particles feel not only the direct interactions, but also the depletion effect caused by the small particles. The mapping can be defined if we fix the number of large particles, $N_{\mathrm{L}}$, and the chemical potential of the small ones, $\mu_{\mathrm{S}}$ (as well as the volume, $V$, and the temperature, $T$ ). The partition function corresponding to this 'semi-grand-canonical' ensemble will then be

$$
Z=\operatorname{tr}_{\mathrm{L}} \operatorname{Tr}_{\mathrm{S}} \exp \left\{-\phi_{\mathrm{LL}}-\phi_{\mathrm{LS}}-\phi_{\mathrm{SS}}\right\}=\operatorname{tr}_{\mathrm{L}} \exp \left\{-\phi_{\mathrm{LL}}-\Omega\right\}
$$

with $\operatorname{tr}_{\mathrm{L}}$ denoting the canonical trace over the large particles, $\operatorname{Tr}_{\mathrm{S}}$ the grand-canonical trace over the small particles and $\phi_{\alpha \gamma}$ the energy of the interaction (in units of $k T$ ) between particles of species $\alpha$ and $\gamma$ (=L, S). The last equality defines the depletion interaction

$$
\Omega \equiv-\ln \operatorname{Tr}_{\mathrm{S}} \exp \left\{-\phi_{\mathrm{LS}}-\phi_{\mathrm{SS}}\right\}
$$

which is a function of the position of the large particles as well as of $V, T$ and $\mu_{\mathrm{S}}$. A diagrammatic expansion of $\Omega$ reveals that it can be written as [14]

$$
\Omega=-V p_{0}+N_{\mathrm{L}} \omega_{1}+\sum_{i<j}^{N_{\mathrm{L}}} \omega_{2}\left(\boldsymbol{R}_{i}-\boldsymbol{R}_{j} ; \mu_{\mathrm{S}}\right)+\cdots
$$

where $p_{0}$ is the pressure of the system when there are no large particles present, $\omega_{1}$ the work needed to insert a single large particle into the system (both terms depend only on $\mu_{\mathrm{S}}$ ) and $\omega_{2}\left(\boldsymbol{R}_{i}-\boldsymbol{R}_{j} ; z\right)$ the work needed to bring together two big particles from infinity to positions $\boldsymbol{R}_{i}$ and $\boldsymbol{R}_{j}$. The dots stand for triplet and higher-order interactions (which are subdominant if the size ratio is small enough).

The function $\omega_{2}$ is the pairwise depletion potential, which is the only relevant contribution in very asymmetric mixtures of hard particles. For small size ratios and low densities of the small particles, it can be estimated as minus the overlap volume between the regions that the large particles exclude the small ones from [4]. Thus it is attractive and short ranged, and its depth is determined by the density of the small particles. Hard spheres interacting with such 'narrow and deep' potentials have been an object of study in recent years, in the context of colloids, and they have revealed a fascinating phase behaviour $[15,16]$. Since van der Waals' work we have known that systems with strong short-range repulsion plus an attractive 
well have a typical phase diagram with vapour, liquid and solid phases. Upon sharpening the well shape, the vapour-liquid critical point shifts to lower pressures (temperatures) until the liquid phase disappears, and at the same time a new critical point arises in the solid region, eventually separating two solids by a condensation transition. Since depletion is one of these potentials, this is the phase behaviour to be expected for a binary mixture upon decreasing the size ratio. In terms of the mixture, a vapour-liquid condensation means a fluid-fluid phase separation; when the liquid disappears, we talk of fluid-solid phase separation (as suggested by the experiments); finally, the solid-solid condensation indicates the phase separation once the big particles have crystallized.

For the latter situation to occur, the range of the depletion well induced by the repulsive core must be smaller than the lattice parameter of the crystal. The volume fraction of the solid phase at the freezing of hard spheres is about 0.54 , corresponding to a lattice parameter of around 1.2 (in diameter units). Thus the diameter ratio must be less than 0.2 for a solid-solid phase separation to exist. Recent simulations of a binary mixture of hard spheres (taken as an effective one-component fluid [14,17] or as the mixture itself [14]) as well as perturbative theories [18] agree with this estimate, finding fluid-solid demixing for diameter ratios above $0.1-0.05$, and fluid-solid as well as solid-solid demixing below this value.

In order to illustrate the phase behaviour of additive hard-core mixtures, we have worked out a density functional theory for parallel hard cubes in the limit of infinite asymmetry $(\epsilon \rightarrow 0$, with $\epsilon$ the diameter ratio). The reason for choosing this 'academic' model is twofold: first, we had previously developed a functional, based on fundamental measures, for mixtures of parallel hard cubes [19], and it turned out to be far simpler than the corresponding one for hard spheres [20,21]; second, depletion in this system is stronger than in a mixture of hard spheres-so much so that the same effect can be obtained for packing fractions of the small cubes $\mathrm{O}(\epsilon)$. The procedure is simple (but involved): we take the free-energy functional for the mixture, $F\left[\rho_{\mathrm{L}}, \rho_{\mathrm{S}}\right]$, and carry out a Legendre transformation to fix $\mu_{\mathrm{S}}$, defining the new functional

$$
\Upsilon\left(\mu_{\mathrm{S}},\left[\rho_{\mathrm{L}}\right]\right)=F\left[\rho_{\mathrm{L}}, \rho_{\mathrm{S}}\right]-\mu_{\mathrm{S}} \int \mathrm{d} \boldsymbol{r} \rho_{\mathrm{S}}
$$

where

$$
\frac{\delta F}{\delta \rho_{\mathrm{S}}}=\mu_{S}
$$

According to (2) we can write

$$
\Upsilon[\rho]=-V p_{0}+N_{\mathrm{L}} \omega_{1}+F_{\text {eff }}[\rho]
$$

which defines the free-energy functional of the effective fluid. We can now take the $\epsilon \rightarrow 0$ limit of $F_{\text {eff }}$ and find (after lengthy calculations)

$$
F_{\text {eff }}[\rho]=F_{\text {PHC }}[\rho]+F_{\text {ad }}[\rho]
$$

where $F_{\mathrm{PHC}}$ is the free-energy functional of a one-component fluid of parallel hard cubes and $F_{\text {ad }}$ arises from the depletion interaction and corresponds to a surface adhesive attraction. This term is proportional to $z \equiv \epsilon^{2} \exp \left(\mu_{\mathrm{S}}\right) / \mathcal{V}_{\mathrm{S}}\left(\mathcal{V}_{\mathrm{S}}\right.$ is the thermal volume of the small cubes), the scaled fugacity of small cubes. Energies are measured in units of $k T$, and volumes in units of the volume of a big cube.

From (5) it follows that the equation of state of the uniform mixture is

$$
p=\rho \frac{1+\rho}{(1-\rho)^{3}}-\frac{3}{2} z \frac{\rho^{2}}{(1-\rho)^{2}} .
$$

It has a van der Waals loop corresponding to a fluid-fluid demixing transition, which is shown in figure 1. That this transition is pre-empted by a fluid-solid demixing is easily seen from the 

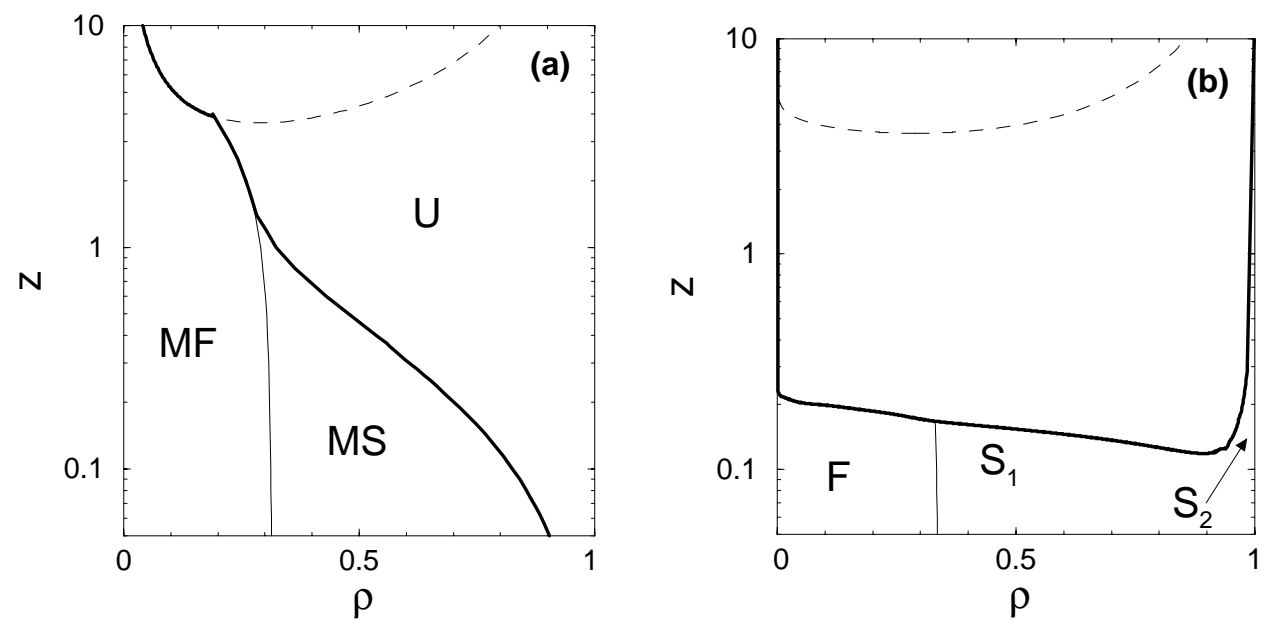

Figure 1. The scaled fugacity of small cubes, $z$, versus the packing fraction of large cubes, $\rho$, for the infinitely asymmetric binary mixture of parallel hard cubes, without (a) and with (b) polydispersity $(\Delta \sigma=0.045)$. (a) The thick line (—) separates the unstable region (U) from the metastable one; the thin line (-) marks the (continuous) transition from a metastable fluid (MF) to a metastable solid (MS); the broken line (-- ) is the fluid-fluid spinodal. (b) The thick line (- - marks the fluid-solid or solid-solid coexistence; the thin line (- ) again marks the (continuous) fluid-solid transition; the broken line $(---)$ is the metastable fluid-fluid coexistence line.

fact that the freezing spinodal (actually the transition, because it is continuous for the fluid of parallel hard cubes [22]) leaves the critical point of demixing in the unstable region. This also holds for any non-zero size ratio [22]. Accordingly, one has to study the solid to produce the phase diagram for this fluid. In doing so, we proceed as usual, parametrizing the density of the solid as

$$
\rho(\boldsymbol{r})=(\alpha / \pi)^{3 / 2} \sum_{\boldsymbol{R}} \exp \left\{-\alpha(\boldsymbol{r}-\boldsymbol{R} d)^{2}\right\}
$$

where the $\boldsymbol{R}$ are the lattice sites of a simple cubic lattice (with lattice parameter 1) and $\alpha$ and $d$ are variational parameters. It turns out that $F_{\text {eff }}[\rho] \rightarrow-\infty$ as $\alpha \rightarrow \infty$ and $d \rightarrow 1^{+}$; i.e. the system collapses to a close-packed solid at any state point of the phase diagram.

This singular phase diagram is not exclusive to this system: it is also found for adhesive hard spheres, as pointed out by Stell some years ago [23]. Simulations of hard spheres with a square-well attraction show the same singular behaviour in the adhesive limit [15]: for narrow and deep potentials there is solid-solid condensation, but as the well range goes to zero and the well depth to infinity, maintaining the second virial coefficient (the adhesive limit), the fluid-solid coexistence region widens to fill up the whole phase diagram.

In spite of this, this formalism allows us to detect metastable phases, which are isolated by large free-energy barriers, the larger the higher $z$. This metastable region exhibits fluid and solid phases, as can be seen in figure 1(a).

In fact, the singularity of this phase behaviour is an artifact of the $\epsilon \rightarrow 0$ limit that can be avoided by introducing some polydispersity in the large particles. If we assume that the cubes have edge lengths chosen at random from a Gaussian distribution of average 1 and standard deviation $\Delta \sigma \ll 1$, then we end up with a free-energy functional very similar to equation (5), but in which no collapse occurs (the details can be found in [22]). The resulting phase diagram depends on the particular value of $\Delta \sigma$, and in the limit $\Delta \sigma \rightarrow 0$ the singular phase diagram 
of figure 1(a) is recovered (the length scale introduced by $\Delta \sigma$ qualitatively produces the same effect as the length of the small particles: see references [14,18]). One of these phase diagrams (for $\Delta \sigma=0.045$ ) is shown in figure 1. The solid-solid demixing is evident from this figure. Also noticeable is the extremely diluted fluid and highly packed solid which coexist in the upper part of the diagram.

As a conclusion to this work, we can say that in about a decade we have passed from questioning the stability of mixtures of hard spheres to a global picture in which demixing is the rule rather than the exception. But demixing has to be understood in a wider sense. On the one hand, we can see from this model that phase separation and freezing are strongly coupled; on the other hand, we learn that the depletion interaction is the key to achieving an understanding of the phase behaviour of very asymmetric mixtures. This fact has renewed the interest in obtaining analytical expressions for these potentials [24], which are useful in driving computer simulations, for instance. On the other hand, we have shown that density functional theory can help us to achieving an understanding of the asymptotic behaviour of such mixtures, connecting them with their corresponding effective fluids. Many other systems and transitions are suitable for undergoing a similar analysis, and we are currently exploring such possibilities.

\section{Acknowledgment}

JAC's work is part of the project PB96-0119 of the Dirección General de Enseñanza Superior (Spain).

\section{References}

[1] Melnyk T W and Sawford B L 1975 Mol. Phys. 29891

[2] Adams D J and McDonald I R 1975 J. Chem. Phys. 631900

[3] Frenkel D and Louis A A 1992 Phys. Rev. Lett. 683363

[4] Asakura S and Oosawa F 1954 J. Chem. Phys. 221255

[5] Lebowitz J L 1964 Phys. Rev. A 133895

Lebowitz J L and Rowlinson J S 1964 J. Chem. Phys. 41133

[6] Biben T and Hansen J-P 1991 Phys. Rev. Lett. 662215

[7] Lekkerkerker H N W and Stroobants A 1993 Physica A 195387

[8] Rosenfeld Y 1994 Phys. Rev. Lett. 723831 Rosenfeld Y 1995 J. Phys. Chem. 992857

[9] Buhot A and Krauth W 1998 Phys. Rev. Lett. 803787

[10] Imhof A and Dhont J K G 1995 Phys. Rev. Lett. 751662

[11] Dinsmore A D, Yodh A G and Pine D J 1995 Phys. Rev. E 524045

[12] Poon W C K and Warren P B 1994 Europhys. Lett. 28513

[13] Caccamo C and Pellicane G 1997 Physica A 235149

[14] Dijkstra M, van Roij R and Evans R 1998 Phys. Rev. Lett. 812268 Dijkstra M, van Roij R and Evans R 1999 Phys. Rev. Lett. 82117 Dijkstra M, van Roij R and Evans R 1999 Phys. Rev. E 595744

[15] Bolhuis P and Frenkel D 1994 Phys. Rev. Lett. 722211 Bolhuis P, Haagen M and Frenkel D 1994 Phys. Rev. E 504880

[16] Tejero C F, Daanoun A, Lekkerkerker H N W and Baus M 1994 Phys. Rev. Lett. 73752 Tejero C F, Daanoun A, Lekkerkerker H N W and Baus M 1995 Phys. Rev. E 51558

[17] Almarza N G and Enciso E 1999 Phys. Rev. E 594426

[18] Velasco E, Navascués G and Mederos L 1999 Preprint

[19] Cuesta J A and Martínez-Ratón Y 1997 Phys. Rev. Lett. 783681 Cuesta J A and Martínez-Ratón Y 1997 J. Chem. Phys. 1076379

[20] Rosenfeld Y, Schmidt M, Löwen H and Tarazona P 1997 J. Phys.: Condens. Matter 8 L577 Rosenfeld Y, Schmidt M, Löwen H and Tarazona P 1997 Phys. Rev. E 554245 
[21] Tarazona P and Rosenfeld Y 1997 Phys. Rev. E 55 R4873

[22] Martínez-Ratón Y and Cuesta J A 1998 Phys. Rev. E 58 R4080

Martínez-Ratón Y and Cuesta J A 1999 J. Chem. Phys. 111317

[23] Stell G 1991 J. Stat. Phys. 631203

[24] Götzelmann B, Evans R and Dietrich S 1998 Phys. Rev. E 576785

Götzelmann B, Roth R, Dietrich S, Dijkstra M and Evans R 1998 Preprint cond-mat/9902189 